\title{
Link-Budget Design and Analysis showing Impulse- based UWB Performance Trade-Off flexibility as Integrator Solution for Different Wireless Short- Range Infrastructures
}

\author{
M.S Jawad, Othman A. R, Z. Zakaria and A.A.lsa. \\ Faculty of Electronics and Computer Engineering \\ (FKEKK), Univeristi Teknikal Malaysia Melaka (UTeM). \\ Hang Tuah Jaya, Durrian Tunggal, Melaka, Malaysia, \\ 76100 .
}

\author{
W. Ismail \\ Kampus Kejuruteraan, Universiti Sains Malaysia, Seri \\ Ampangan, 14300 Nibong Tebal, Seberang Perai Selatan, \\ Pulau, Pinang, Malaysia.
}

\begin{abstract}
Future wireless indoor scenarios are expected to be complex requiring wireless nodes to adaptive responding to dynamic changes according to channel conditions. Interacting with neighboring nodes to achieve optimized performance in term of date-rates, distance and BER performance are the main concerns of designing future wireless solutions. IR-UWB came to the picture as the missing Puzzle to achieve these requirements and gluing the different wireless indoor existing infrastructures in global platform solutions. This paper shows the flexibility of IR-UWB in signal design at the physical layer level as cross-layer architecture of optimized performance. A detailed performance analysis presented in this paper as a mathematical model of the proposed wireless solution and described in proposed link budget design template. The performance evaluation is carried-on to show the proposed system as a good candidate in different wireless scenarios for different data-rate requirements, distance for specific requirement BER. Simulations results and well as experimental statistical analysis of the received signal under different channel models and conditions are carried-out as a proof of concept of the proposed system.
\end{abstract}

Keywords-Ultra Wideband; Time Hopping-pulse position Modulation; Radio Frequency Identification; Wireless Sensors Networks; RAKE Receiver; Bit Error rate

\section{INTRODUCTION}

The impulse based IR-UWB technology has attracted a great deal of research recently, due to its enormous advantages which can be utilized for wireless indoor applications and infrastructure such as RFID and WSN networks. Thanks to its signal' unique physical characteristics which can be described as a very short a train of pulses in ultra-wide bandwidth, making this type of signal a very low duty cycle in comparison with classical narrowband signal. This will add a good advantage of IR-UWB as reduction in power consumption, since in these discontinuous emitted pulses the emitter will consume much less.[1] This type of continuous emission technique can be used with a large bandwidth to achieve a high data [2]. The IR-UWB as a discontinuous emission technique in a wide bandwidth can be seen as a noise-like signal difficult for any intruder to intercept or detect making this signal secure at the media with good performances as the ability to co-existence with other narrowband technologies and resist to interface. Also, a high resolution for localization can be achieved using this technique of low-duty cycle pulses in wide bandwidth allowing the tracking of objects with less than $1 \mathrm{~cm}$ accuracy. As this technology considered carrier less technology with no need for carrier signal as modulation done by altering the pulses positions, amplitude or polarity so the IR-UWB emitter can be designed without RF stage. With no VCO (Voltage Control Oscillator), mixer and other RF components this mostly digital architecture will consume less power and allow simple receiver structure [3-4]. All these advantages can be utilized for wireless indoor sensing and identification applications combining the functionalities of detecting, identifying and tracking the objects in one global platform. Since RFID and WSNs communication systems share in common some system design constrains such as the limitations of power consumption and hardware complexity[5] . IR-UWB can be a good integrator for both of them. Fortunately many IR-UWB signal parameters can be adjusted and tuned allowing what's called a reconfigurable IR-UWB radio[4]. A link budget analysis model is proposed to show system parameters selection and then the radio performance to understand the trade-off flexibility for different data- rates, ranges (between the transmitter and receiver) and BER performance. The proposed model can be utilized for different high-rate, low-rate, and short to medium wireless indoor scenarios. The structure of the paper is as follows. Section2 consider a literature review studies to show the utilization of IR-UWB for different high-rate and low-rate WPAN with the focus on wireless sensing and identification. Section 3 models mathematically the IR-UWB transmission chain to fully understand the signal generation parameters. Section 4 shows a suitable IR-UWB receiver structure and its performance in AWGN channel model, then in section 5 the link budget with different signal physical parameters is modeled and analyzed for a given IR-UWB power compliant with the FCC rules [6] and with some simplified hypotheses to show the maximum distances of propagation when a pre-determined probability of error must be guaranteed for a given data-rate at the receiver. Finally, the results remarks are concluded with suggestions for future researches. 


\section{LITERATURE STUDIES}

Many researches in literature proposing the utilization of IR-UWB in WPAN (Wireless Personal Area Network) communication for high data-rate, low data-rate and from short to medium ranges applications [7] . (Pezzin, M., B. Denis, et al) they propose utilization of IR-UWB in low complexity and low data rate platform for location and tracking services [8]. Achieving security by making the wireless signal secure physically at the medium rather than achieving security by securing the contents of data using some cryptography algorithm can be utilized for RFID system using IR-UWB technique and this security approach can reduce the hardware complexity significantly especially for passive RFID tags by eliminating the logic gates ( at the hardware level) needed to cipher or decipher blocks of data and this been proposed recently in some researches [9-10]. Also Time Hoping multi-Pulse Position Modulation (UWBTH-MPPM) is been proposed for high data rate wireless short ranges applications[2].

Many proposals been introduced in literature with the aim of integrating both RFID and WSN networks to combine their capabilities of detecting the objects of interest and tracking these objects while sensing, collecting and processing the data [11-14] and some researches utilizing the great advantages of IR-UWB as integrator for both RFID and WSN systems [15]. IR-UWB technology also has the potentials to support identifications, Location, sensing and connectivity in ubiquitous computing environments and in cognitive radio scenarios since this technology consider good candidate satisfying many requirements of cognitive radio[16-17]. The IR-UWB reconfiguration flexibility in many aspects is been studied with the link budget analysis in [4]. As a conclusion from all these previous studies, it clearly that the IR-UWB unique characteristics with the reconfiguration capabilities of IR-UWB can be utilized for many wireless indoor applications to show the trade-off flexibility for different data-rates, ranges and BER requirements and the best way to analyze this flexibility is by proposing link budget analysis model which this paper investigate.

\section{IR-UwB MODEL AND PARAMETERS}

The time hopping as spectrum spreading technique allowing multiple access performance with the M-ary Pulse Position modulation is considered due to its simplicity and suitability for simple RFID tags or wireless nodes sensors. The signal generated with time hopping (TH) with M-ary with pulse position modulation for K-th user can be mathematically modeled as

$$
\begin{gathered}
S^{K}(t)=\sum_{j=-\infty}^{\infty} A_{d[J / N s]}^{(k)} p\left(t-T_{f}-C_{j}^{(k)}-\right. \\
\left.\delta_{d}(k)_{[J / N s]}\right) \ldots \ldots \ldots \text { (1) [18] }
\end{gathered}
$$

Understanding the signal parameters with their typical values is important to show the flexibility and reconfiguration capabilities of IR-UWB at the physical layer. These parameters can be described as the following:

$\mathrm{P}(\mathrm{t})$ represents the pulse shaping which is the second derivative of Gaussian pulse with pulse width $T_{p}, T_{f}$ is the rame time and each frame is divided into $N_{h}$ time slots with duration time $T_{c} . C_{j}^{(K)}$ is the pulse shaping patterns, which is pseudorandom numbers with period $T_{c}$, it represent the time hopping sequence for $K_{T h}$ users, here additional shift is required to avoid catastrophic collisions caused by the multiple access interference (MAI). The d parameter is the sequence for the data stream generated by the $K_{T h}$ user after channel coding. $\delta$ is the shift introduced for pulse position modulation utilized by M-ary PPM. If it is assumed that the signal amplitude $A^{(v)}=1$, for the M-ary PPM signal, then equation (1) can be written as $S^{(v)}(t)=\sum_{j=-\infty}^{\infty} p\left(t-j T_{f}-\right.$ $c_{j}^{(k)} T_{c}-\delta d_{[j / N s]}^{(k)}$. The following figures show the simulation of the generated signal then the PPM modulation for transmitting 1010 code.
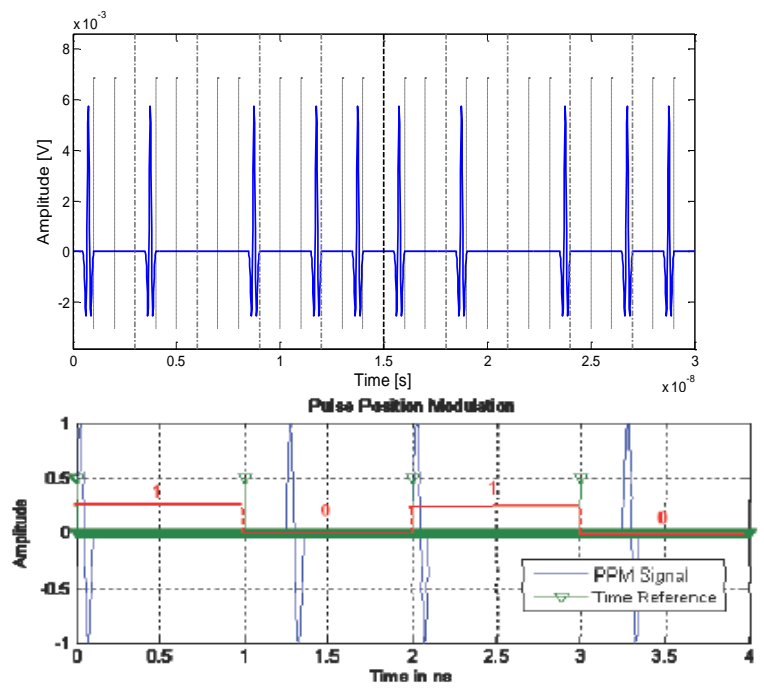

Fig. 1. a:Simulation of TH-PPM signal generation.

Fig. 1. b: TH-PPM signal coding

\section{A. IR-UWB Receiver structure}

Optimum receiver structure in AWGN channel

For M-arry PPM, assuming the time shift $\xi$ introduced by PPM modulation is larger than the pulse duration $T_{m}$, as shown in the Figure-3- where the structure of the optimum receiver in this case, shows that the output of the signal correlators will be considered as decision variable and can be expressed as the following :-

$$
Z_{0}=\alpha S_{m 0}+n_{0}
$$$$
Z_{M-1}=\alpha S_{m(M-1)}+n_{M-1}
$$

Where $S_{m K=\sqrt{E_{T X}}} \int_{0}^{T_{S}} P_{0}(t-m \xi) P_{0}(t-k \xi) d t$

$P_{0}(t)$ is the energy- normalized waveform of the basic pulse, $E_{T X}$ is the transmitted energy per pulse, $\xi$ shift introduced by PPM modulation. [8] [7] 


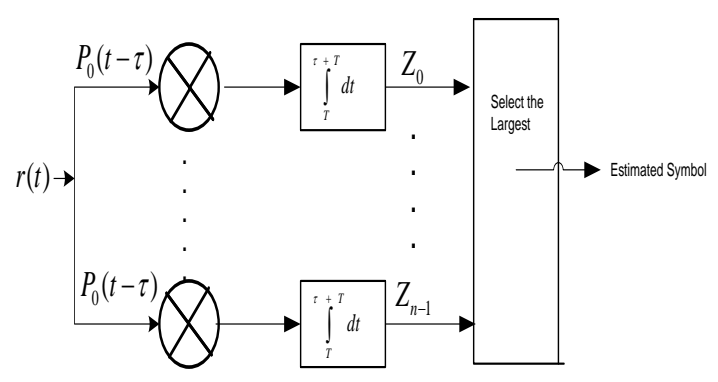

Fig. 2. TH-PPM receiver structure for AWGN channel

When $\mathrm{M}$ possible waveforms are generated, the average error probability on symbol $P_{r e}$ is the probability of misdetection one of the symbols, the error occurs at the receiver side if at least one $M-1$ output $Z_{K}$ is larger than $Z_{0}$, the probability of this even to occur can be expressed as the following :-

$\operatorname{Pr}_{e}=\frac{1}{\sqrt{2 \pi}} \int_{-\infty}^{+\infty}\left(1-\left(\frac{1}{2 \pi} \int_{-\infty}^{y} e^{\frac{-x^{2}}{2}} d x\right)^{M-1}\right) e^{\left[\frac{-1}{2}\left(y-\sqrt{\frac{2 E_{R x}}{N_{0}}}\right)^{2}\right]} d y$ (2) $[18]$

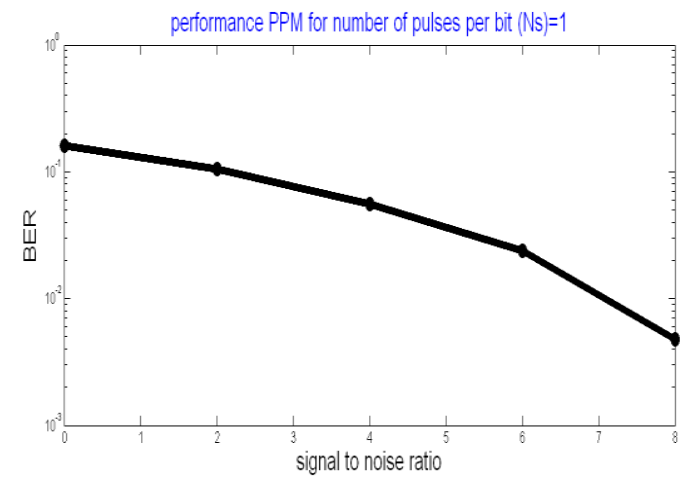

Fig. 3. TH-PPM receiver performance

\section{B. Receiver Structure in multipath fading environments}

To optimize correlation in multipath environment, additional correlators should be associated for different replicas of the same transmitted waveform, this scheme what is called rake receiver was invented by (Prince and Green, 1958)

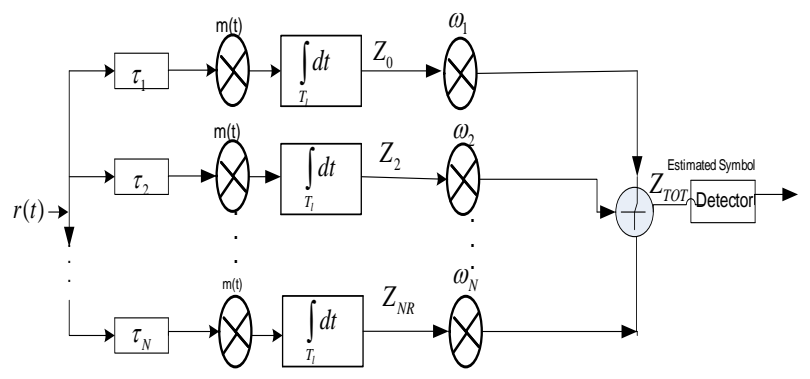

Fig. 4. RAKE receiver structure in Multi-path fading channel
As shown in Figure 4, the RAKE receiver consists of parallel bank of $N_{R}$ correlators, where each correlator is associated to one of the different replicas of the transmitted pulse, so that the correlator mask $m \mathrm{j}(\mathrm{t})$ on the time $\mathrm{j}$-th branch of the RAKE is aligned in time with the $\mathrm{j}$-th delayed replica of the transmitted pulse $m_{j}(t)=m\left(t-\tau_{j}\right)$, where $\mathrm{m}(\mathrm{t})$ is the correlation mask, and $\tau_{j}$ is the propagation delay of the $j$-th path, at the end the output of the correlators feeding the combiner. The weighting factors $\left(\omega_{1}, \ldots ., \omega_{N r}\right)$, can be combined depending on the diversity method implemented in the receiver. In MRC case, to output of each branch is multiplied by the weighting factor, which is proportional to the signal amplitude on that branch

\section{LINK BUdGET ANALYSIS}

In reality UWB radio signals, coexist with other radio signals which causes interferences with other UWB or narrowband communication systems it's also subjected to channel conditions changes especially in severe multipath environments. The problem is for any UWB system optimization should be within the regulated values of intrinsic UWB principle.

As such, UWB should be flexible to adapted to environments, whether this refer to channel condition changes or interference patterns while maintaining compatibility with the regulations on emitted UWB signal . The UWB signal format offers interesting and appealing properties by high numbers of tunable parameters and this is especially due to the impulsive nature of the pulses. Signal characteristics can be tuned efficiently by playing with a variety of parameters. These parameters include the transmission factor such as the number of pulses representing one bit, coding factors such as periodicity and cardinality of TH. Modulation factors related to the PPM shift, shaping factors related to specific pulse shape.

\section{A. Adjusting emitted radiation}

The power limitations of UWB signal set by FFC emission masks determine the maximum allowed transmitted power. In order to understand how to read an emission mask, it is important at the beginning to define the effective isotropic radiated power (EIRP) for a given range of operating frequencies. EIRP can be understood as the maximum power that the transmitter can transfer to the transmitter antenna, so it can be defined as the product of the available power at the transmitter $W_{T x}$, expressed in Watts and the gain of the transmitter antenna $G_{A T}$.

$$
E I R P=W_{T X} G_{A T}
$$

The condition here is the maximum power transfer from the output impedance of the transmitter $Z_{T X}$ to the input impedance of the antenna $Z_{A T}$ is verified that is, $Z_{A T}=$ $Z_{T X}$. EIRP is measured in $\mathrm{dBm}$ that is10 $\log _{10}$ EIRP mWatts.

So in brief, emission mask impose limits on the PSD of the emitted signal and can be expressed as $\mathrm{dBm} / \mathrm{Hz}$ or $\mathrm{dBm} / \mathrm{MHz}$. 
commonly provided in practice, the emission mask expressed in term of power value for a given frequencies which indicate the maximum allowed radiated power with measured bandwidth $(\mathrm{mb})$ around center frequency $f_{C}$.

\section{B. IR-UWB Link Budget Analysis over AWGN Channel}

Given allowed power and under simplified hypotheses, we can evaluate the maximum distance over which the propagation can reach, with a predetermined level of error should be guaranteed at the receiver for a given data rate.

The analysis start by observing the received energy $\mathrm{E}$ over a finite time interval, allowing the decision at the receiver and in case of propagation over AWGN channel, the received energy is based on the signal term $E_{r}$ and the thermal noise $E_{\text {noise }}$, from the signal to noise ratio SNR can be defined as:

$$
\mathrm{SNR}=\frac{E_{r}}{E_{\text {noise }}} \geq M . S N R_{\text {spec }}
$$

Where $S N R_{\text {spec }}$ the required SNR, M is the system margin

So the required energy at the receiver can be expressed:

$$
E_{r=} M . S N R_{\text {spec }} \cdot E_{\text {noise }} \cdots . .(5)
$$

The thermal noise energy can be defined as:

$$
E_{\text {noise }}=\frac{1}{2} N_{o}=\frac{1}{2} K T \text { Temp }
$$

Where $N_{o}$ is Single-sided thermal noise spectral power density, $\mathrm{K}$ is the Bolzman constant and Temp $p_{S}$ the spot noise temperature.

$$
\operatorname{Temp}_{s}=\operatorname{Temp}_{A}+(F(f)-1) \text { Temp }_{o}
$$

Where $T e m p_{A}$ is the receiving antenna temperature, $F(f)$ is the spot noise figure and Temp $p_{o}$ is the standard temperature $\left(290^{\circ} \mathrm{k}\right)$

$E_{r}$ Can be defined as received signal power $P_{r}$ over a finite time interval $T_{b}$, so from equation (2) $E_{r}$ can be expressed as $E_{r}=M \cdot S N R_{\text {spec }} \cdot \frac{1}{2} K\left(\right.$ Temp $_{A}+(F(f)-1)$ Temp $_{o}$

$$
\begin{gathered}
\text { Which is equivalent to } \\
P_{r} T_{b}=M \cdot S N R_{S p e c} \cdot \frac{1}{2} K\left(\operatorname{Temp}_{A}+(F(f)-1) \text { Temp }_{o}\right) \ldots .(9) \\
\text { The receiver power } P_{r}=2 \int_{f_{l}}^{f_{H}} \frac{P_{s(f)}}{A_{F S}} d f=2 \int_{f L}^{f H} \frac{P_{S(f)}}{\frac{(4 \pi)^{2} D^{2} f^{2}}{G_{T} G_{R} C^{2}}}
\end{gathered}
$$

$P_{S}(f)$ Is the double sided power spectral density, $A_{F S}(f)$ is the free space attenuation.

So equation (6) can be expressed as:

$$
2 T_{b} \int_{f L}^{f H} \frac{P_{s(f)}}{\frac{(4 \pi)^{2} D^{2} f^{2}}{G_{T} G_{R} C^{2}}} d f=
$$

$$
\text { M.SNR } R_{\text {Spec }} \cdot \frac{1}{2} K\left(\text { Temp }_{A}+(F(f)-1) \text { Temp }_{o}\right)
$$

From equation (8) we can derive the squared value of the maximum distance $\mathrm{D}$ that can be covered by transmission.

$$
D^{2}=\frac{\frac{G_{T} G_{R} c^{2}}{(4 \pi)^{2}} 2 T_{b} \int_{f_{L}}^{f_{H}} \frac{P_{S}(f)}{f^{2}} d f}{M_{S} S N R_{\text {Spec }} \cdot \frac{1}{2} K\left(\text { Temp }_{A}+(F(f)-1) \text { Temp }_{o}\right)} .
$$

From equation (9) we can derive the maximum distance of propagation of an UWB point to point link given $P_{\text {smin }}$ and the given target $S N R_{\text {Spec }}$

For the sake of simplicity it can be assumed that emp $_{A}=$ Temp $p_{o}$, in this assumption, the noise temperature at the receiving antenna is considered the standard temperature. This assumption is practical for terrestrial links. Equation (9) becomes:

$$
D^{2}=\frac{\frac{G_{T} G_{R} c^{2}}{(4 \pi)^{2}} 2 T_{b} \int_{f_{L}}^{f_{H}} \frac{P_{S}(f)}{f^{2}} d f}{M_{S} S N R_{\text {Spec }} \cdot \frac{1}{2} F_{k} \text { Temp }_{o}}
$$

In the worst case for $P_{S}(f)$, it can be substituted by its lower case value $P_{\min }$ within its bandwidth, so equation (10) can be then:

$$
D^{2}=\frac{\frac{G_{T} G_{R} c^{2}}{(4 \pi)^{2}} 2 T_{b} P_{S_{M i n}} \int_{f_{L}}^{f_{H}} \frac{P_{S}(f)}{f^{2}} d f}{M_{S} S N R_{\text {Spec }} \cdot \frac{1}{2} F_{k} \text { Temp }_{o}} \ldots \ldots \ldots
$$

And it can be expressed as in equation (12):

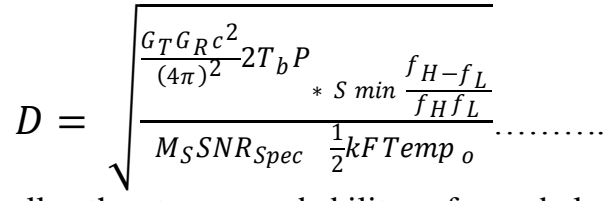

Usually the term, probability of symbol error $P_{r e}$ is commonly used for system specifications, rather than SNR. Modulation scheme effecting the relation between SNR and $P_{r e}$ and that can be easily expressed in term of noise in AWGN. In case of optimum AWGN receiver, the performance can be evaluated for various modulation types as the following:

Given a target $P_{r e}$, we want to evaluate the required $S N R_{\text {Spec }}$, we assume the system margin is zero for $\left(M_{s}=0\right)$ for the sake of simplicity. referring to some resources of digital communication dealing with these relations for different modulations schemes (lee and Messecrschmitt (1994), Proakis (1995) and summary in Guvence and Arslan (2003)), we can summarize the link budget analysis for M-PAM and M-PPM as the following:

First: for M-ary PAM

$$
\begin{aligned}
& P r_{e}=\left\{1-\frac{1}{M}\right\} \operatorname{erfc}(y) \\
& \text { Where } y^{2}=\frac{S N R_{\text {Spec }}}{\frac{2}{3}\left(M^{2}-1\right)}=\frac{P_{r} T_{b} \log _{2} M}{\frac{1}{2} k F \operatorname{Temp}_{o} \frac{2}{3}\left(M^{2}-1\right)}= \\
& \frac{E_{b}}{N_{o}} \frac{3 \log _{2} M}{N o\left(M^{2}-1\right)} \text {. }
\end{aligned}
$$

With SNR is sufficiently high $\left(\frac{E_{b}}{N_{o}}>4.43 \mathrm{~dB}\right)$ then the upper bound for M-ary orthogonal PPM for $\frac{E_{b}}{N_{o}}>4.43 \mathrm{~dB}$ can be expressed as :

$$
\operatorname{Pr}_{e}<e^{-\log M\left(\frac{E_{b}}{N_{o}}-2 \log _{e} 2\right) / 2}
$$

Equation (15) is valid only for high SNR values and for SNR vales lower than $4.43 \mathrm{~dB}$, the lower bound can be expressed as $P r_{e}<2 e^{-\log _{2} M\left(\sqrt{E_{b} / N_{o}}-\sqrt{\left.\log _{e} 2\right)}\right.}$ 
Figure (5) evaluate the $P r_{e}$ for an M-ary PAM signal and the upper bound of the $P r_{e}$ for M-ary PPM .

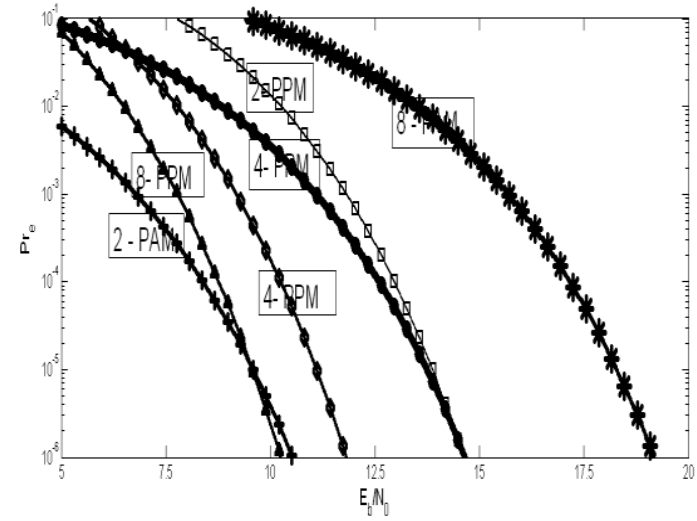

Fig. 5. comparison for probabilities of symbol error for M-PAM and PPM according to equations $18 \& 19$

The notice here is that the performance improves as $\mathrm{M}$ increases for the case of M-PPM signal, whereas in case of MPAM the opposite is true.

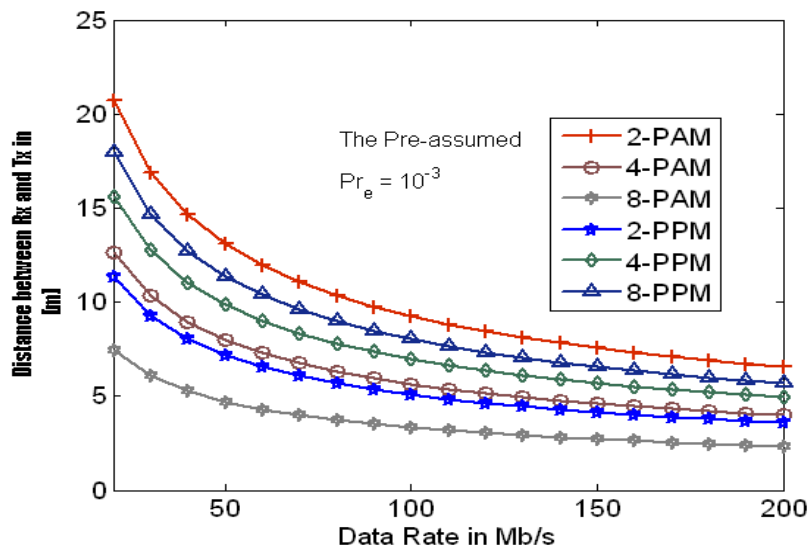

Fig. 6. Trade-off Flexibility for the distance between transmitter and receiver as a function of data-rate between M-PAM and M-PPPM signal

\section{REAL-Time Simulation OF IR-Uwb COMMUNiCATION SYSTEM}

Simulink fixed-point simulation model been carried-out showing the real-time point-to-point IR-UWB communication behavior for different signal parameters propagating over multi-path IEEE 803.15.3a channel model that can simulate the signal behavior over different LOS (lines of Sight) channels types and NLOS (Non-line of Sight) with different distances ranging from CM1 to CM2 channel, then simulation of the received signal will show the effect of the multipath fading reflection on the channel. At the end the BER performance evaluation of the transmitted signal at the receiver side will show in real-time the signal behavior and its trade-off flexibility of the performance for different sets of the signal parameters (which directly indicating the data-rates) and the distances from different multi-path channel types.

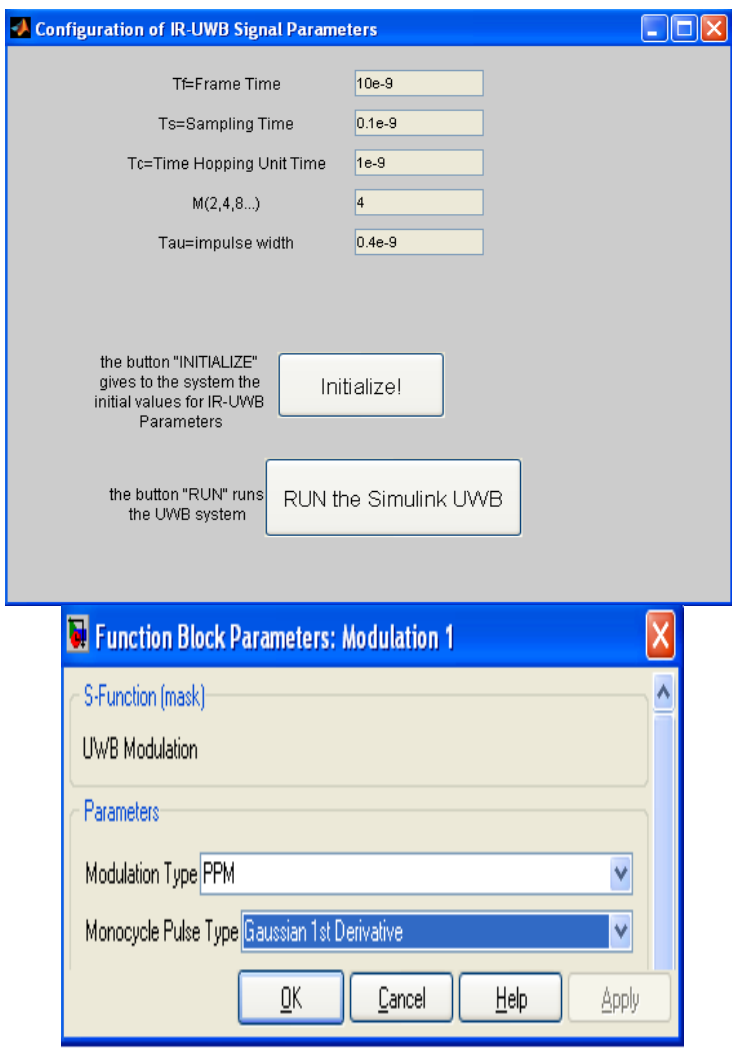

Fig. 7. Parameters Settings for TH-PPM Signal

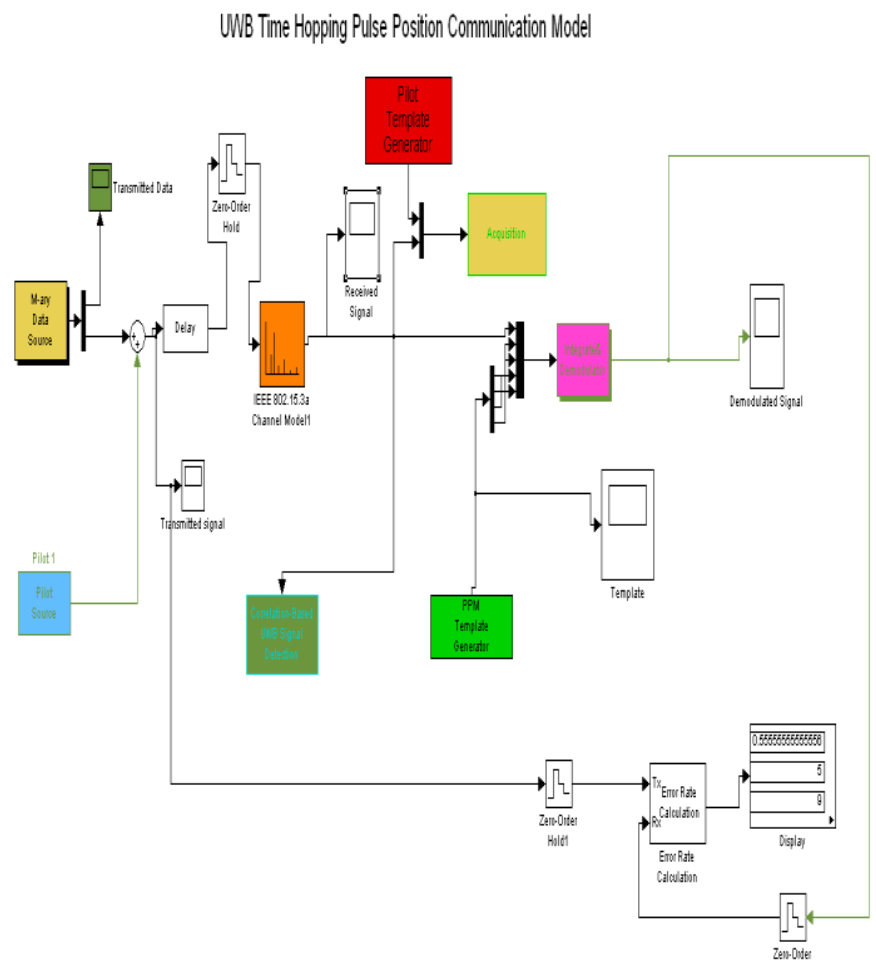

Fig. 8. IR-UWB Communication Model with BER Performance Evaluation 


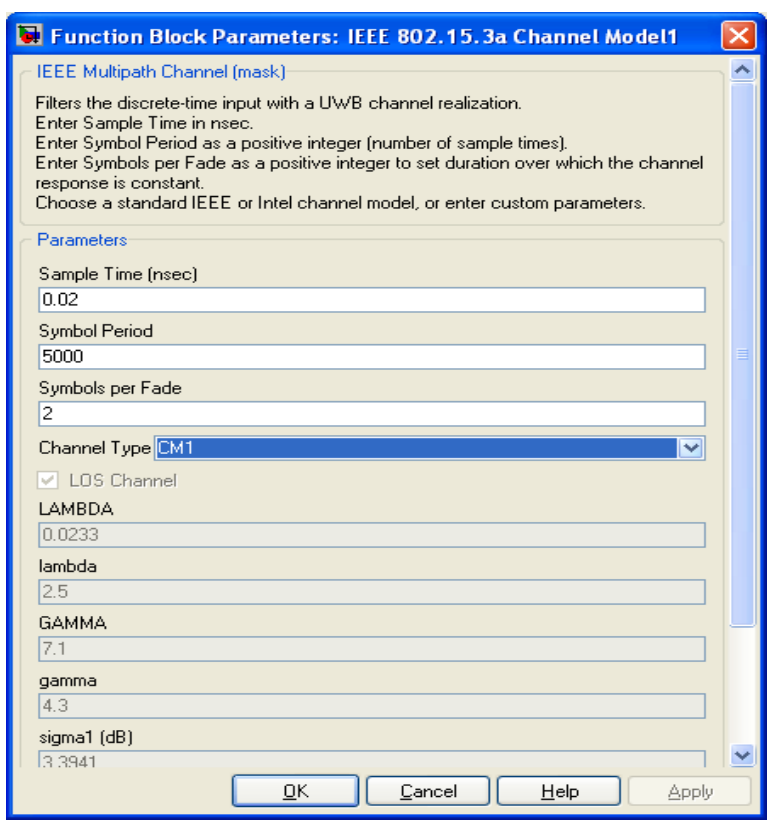

Fig. 9. Configurations of IEEE 803.15.3a Channel Model

Table I shows the system parameters in real-time implementation as well as the BER results. All values are obtained from the Simulink workspace during and after system simulation. The IR-TH PPM system is designed for specific data rates of predefined BER requirements propagating over four IEEE 802.153a channel model scenarios (C1 to C4), as shown from figure 4.19. The required BER should be within the range of $1 \times 10^{-2} \mathrm{~s}$ to $4 \times 10^{-2} \mathrm{~s}$. The design of the system is according to the settings of these parameters, including signal generation, shaping (at the data-source level), and modulation (at the signal-pilot level), to achieve the required performance of the radio link, given that the BER result is $2 \times 10^{-2} \mathrm{~s}$.

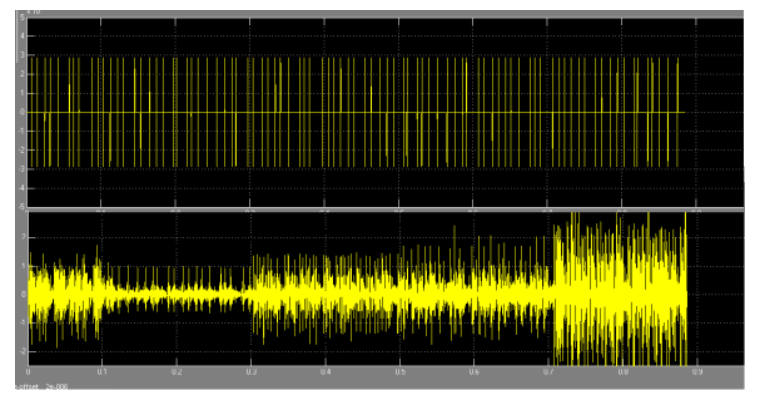

Fig. 10. the upper part is the TH-PPM Transmitted signal propagating over IEEE 802.15.3a channel model with configurations as stated in figures $7 \& 9$. The lower part is the received signal with the multi-path channel model effects.
TABLE I. IR-Uwb SYSTEM PARAMETERS AND EVALUATION IN REAL TIME

\begin{tabular}{|c|c|}
\hline \multicolumn{2}{|l|}{ Data source } \\
\hline M-ary & 2 \\
\hline Symbol time & $100.000 \mathrm{~ns}$ \\
\hline Link selector number [0 reserved for pilot] & 1 \\
\hline Promised data-loading state & {$[11000]$} \\
\hline \multicolumn{2}{|l|}{ Modulation } \\
\hline Frame time & $10.000 \mathrm{~ns}$ \\
\hline TH unit time & $1.000 \mathrm{~ns}$ \\
\hline PPM unit time & $0.156 \mathrm{~ns}$ \\
\hline Impulse width & $0.2877 \mathrm{~ns}$ \\
\hline Symbol time in terms of frame time $\mathrm{T} / \mathrm{Tf}$ & 10 \\
\hline PN sequence period & 31 \\
\hline Average signal power & $-40.0 \mathrm{dBm}$ \\
\hline Pulse energy & $1.000000 \mathrm{e}-015 \mathrm{~J}$ \\
\hline Initial state of PN code shift register $[\mathrm{m}=5]$ & [ 10001$]$ \\
\hline Bits used to generate hopping code & 3 \\
\hline \multicolumn{2}{|l|}{ Channel } \\
\hline \multicolumn{2}{|c|}{ IEEE 802.15.3a channel model } \\
\hline UWB signal detection threshold & $95 \%$ \\
\hline Acquisition threshold & $70 \%$ \\
\hline False alarm threshold & $30 \%$ \\
\hline Initial state of receiver PN code shift register & [00010] \\
\hline \multicolumn{2}{|c|}{ Simulation in real time } \\
\hline
\end{tabular}

Sampling time (uwb.Ts) $=0.0500 \mathrm{~ns}$; UWB signal detected; time $=4.550 \mathrm{~ns}$ coefficient $=97.515$

Acquisition coefficient $=10.092 \%$

Acquisition coefficient $=-3.805 \%$

Acquisition coefficient $=100.319 \%$

PN hopping code acquisition

Acquired frame time $=590.000 \mathrm{~ns}$

Tracking coefficient $=101.779 \%$

Tracking coefficient $=100.507 \%$

Data synchronization; demodulation begins.

Demodulation start time $=810.000 \mathrm{n}$

\begin{tabular}{|c|c|}
\hline BER command & Bercal \\
\hline BER result & $\mathbf{0 . 0 2 0 0 0 0 0 0 0 0 0 0 0 0 0} \mathbf{s}$ \\
\hline
\end{tabular}

VI. FunCtional COMPARISON OF Th-PPM

COMMUNication Using P220 Evaluation Kit

This section provides a meaningful comparison among different TH-UWB approaches and their performance metrics. A functional comparison approach is adopted. First, a number of different application scenarios are considered. Then, performance metrics pertaining to these application scenarios are used for comparison. Finally, conclusions describing the performances according to indoor and outdoor environments and recommendations for applications are drawn. 
The deployment in WPAN communication scenarios is considered for both indoor and outdoor environments, wherein data rate varies from very high to low for different coverage ranges. These scenarios are applicable in wireless body networks, in which sensor nodes are all placed in the field of the human body and require radiating extremely high-data-rate UWB pulses for imaging, as well as in UWB radar applications for monitoring patient movements in intensive care units, which can be classified as high rate with shortrange WPAN (few meters). Low data rates with medium range are applicable for RFID systems and WSNs, whereas high data rates with medium range are applicable in digital multimedia smart environments (including homes, clinics, and libraries), where nodes send high-data-rate multimedia contents.

\section{A. Propagation Environments}

This subsection presents the results of the measurements conducted in selected indoor and outdoor environments under the following conditions.

- The outdoor environment is an open grassy plain under LOS condition for transmitter and receiver positioning, as shown in Figure 11.

- The indoor environment is under NLOS conditions, wherein numerous thick and thin walls, electrical equipment, and the distances between the transmitters are considered. The layout of this test site is shown in Figure 11, where T1 represents fixed transmitter location; and R1, R2, R3, and R4 denote different receiver distances from the transmitter location. The estimated ranges between the four positions of the transmitter and the receiver are: $30.48 \mathrm{~m}, 38,1 \mathrm{~m}, 45.72$ $\mathrm{m}$ and $60.96 \mathrm{~m}$.

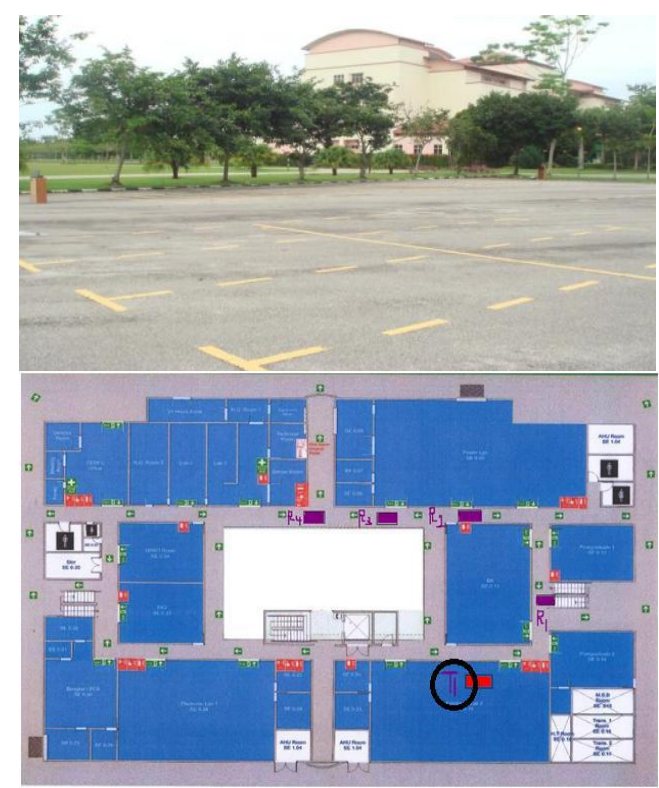

Fig. 11. Experimental layout of indoor/outdoor propagation

\section{B. Experimental Results and Discussion}

Figures 12 and 13 show variations of bit energy to effective noise density ratio $\mathrm{Eb} / \mathrm{Neff}$ as a function of distance and data rates between the transmitter and the receiver based on the measurements for four transmission rates in two conducted sets of measurements (indoor and outdoor) for short-to-medium-range communications. Based on the result, $\mathrm{Eb} / \mathrm{Neff}$ decreases as distance increases of increased data rates for both outdoor and indoor experimental environments. For example, at $9.6 \mathrm{Mbps}$ the Eb/Neff is less than $5 \mathrm{~dB}$ which is the lowest compared to other lower data rates with the same distance. This is because as the data rates become higher, more data can be transmitted which makes more noise can disturb into the system data.

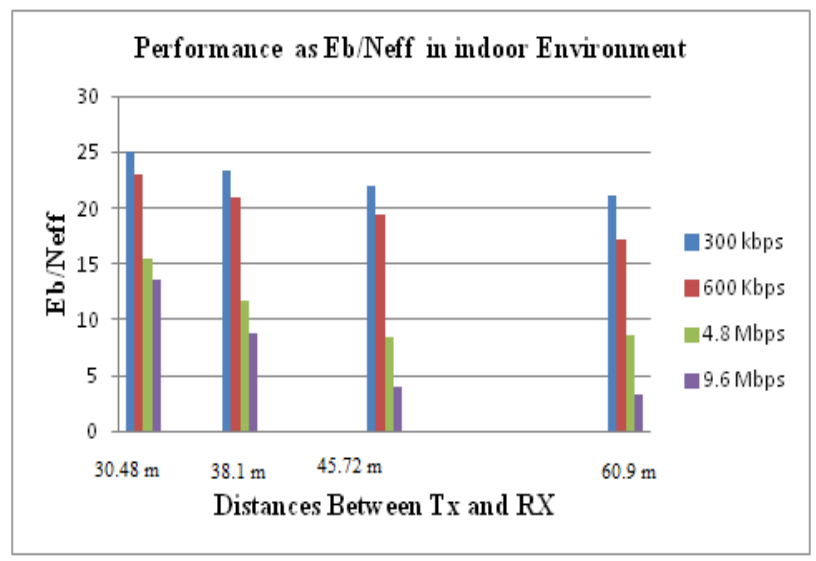

Fig. 12. Performance evaluations with regard to Eb/Neff in indoor environment

In comparison with indoor performance, outdoor outperform indoor in general as shown in Figure 13 for many cases for example; Eb/Neff for $300 \mathrm{Kbps}$ for all distances, 4.8 Mbps in $38.1 \mathrm{~m}$ and $60.96 \mathrm{~m}$ also 9.6 at $45.72 \mathrm{~m}$. This can be explained as the pulses in indoor environment are more exposed to multipath fading effects which resulting in pulses power loss.

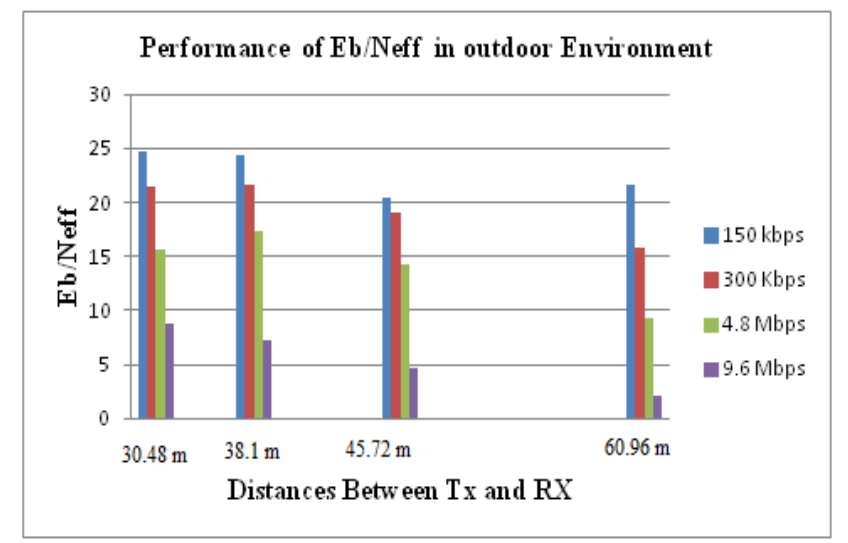

Fig. 13. Performance evaluations with regard to Eb/Neff in outdoor environment

BER obtained at different data rates for indoor and outdoor measurements are respectively plotted in Figures 14 and 15. It shows that in the extreme distance case of $60.96 \mathrm{~m}$ in indoor environment, the BER is higher for all the data rates and for the extreme data rate of $9.6 \mathrm{Mbps}$ at the distance of $54.72 \mathrm{~m}$ the BER also is with higher value for indoor compared to outdoor performance. Results in general show that BER increases as distance and data rate increase and this is in 
agreement with the theoretical analysis and previous MATLAB simulation. Similarly, the performance of IR-UWB in outdoor scenarios with regard to BER in various distances and data rates outperforms that in indoor scenarios especially for the cases of high data rates with longer distances.

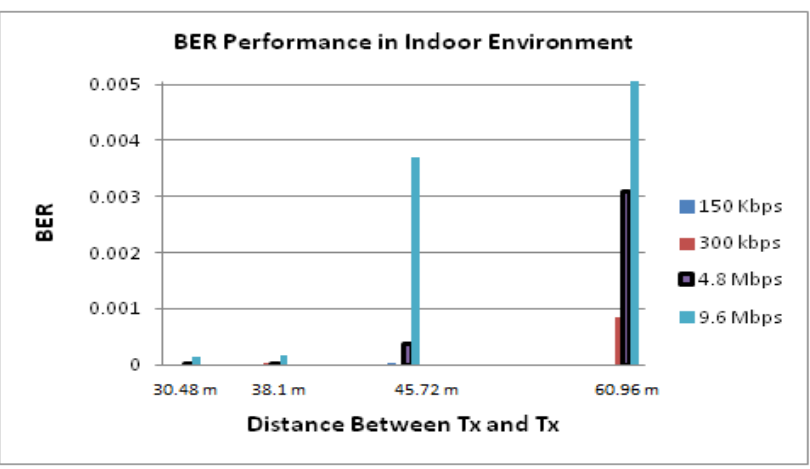

Fig. 14. Performance evaluations with regard to BER in indoor environment

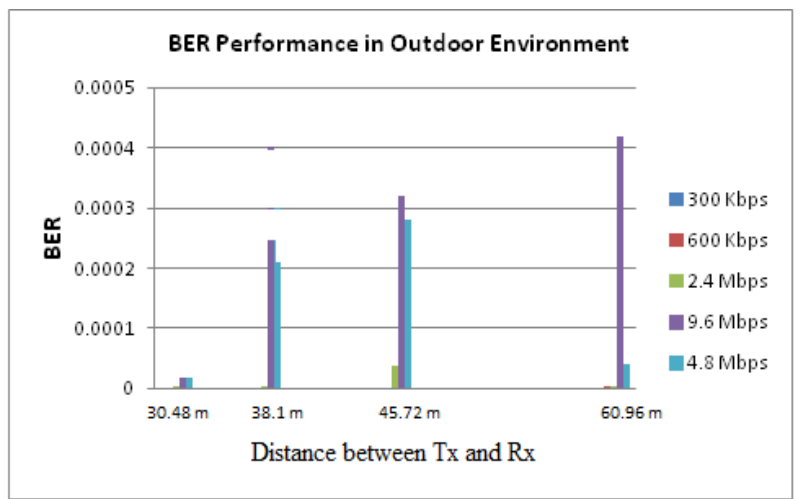

Fig. 15. Performance evaluations with regard to BER in outdoor environment

Figures 16 and 17 plot the experimental relationship between BER and Eb/Neff for outdoor and indoor environments, respectively. Based on the curves, the performance generally matches with the theory. However, results differ significantly in several cases of the experiment in outdoor environments at data rates between $4.8 \mathrm{Mbps}$ and 9.6 Mbps (Figure 17). Such difference can be related to severe multipath degradation in several locations where conditions change, as well as to the limitation and sensitivity of the evaluation tool-kit.

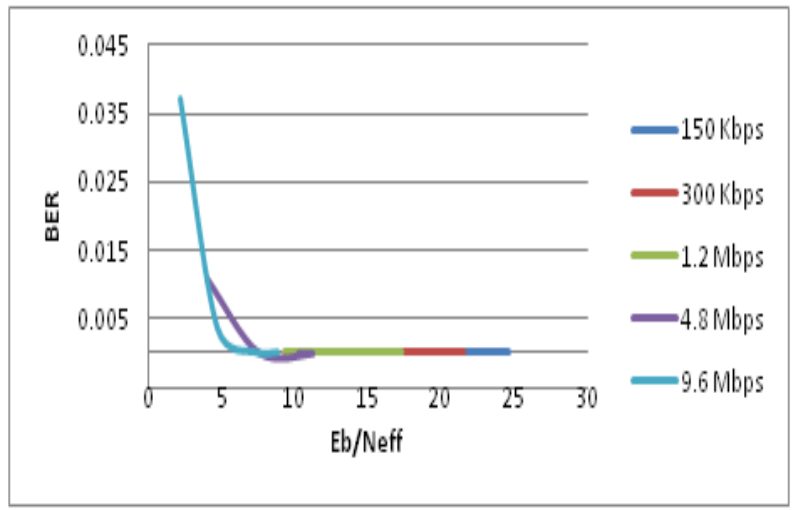

Fig. 16. Comparison of performance evaluation between BER and Eb/Neff for indoor environment

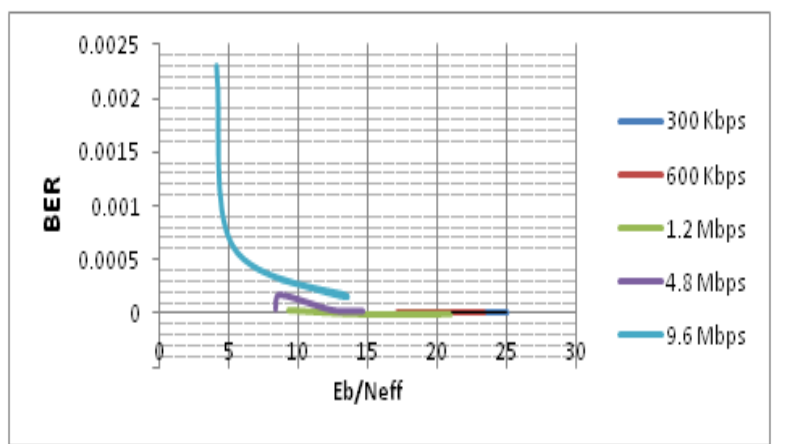

Fig. 17. Comparison of performance evaluation between BER and Eb/Neff for outdoor environment

The achieved link throughput is effective evaluation criteria for wireless solution designer. The tunable impulse based UWB proposed solution is evaluated experimentally for the actual achieved data rate in different indoor distances as shown in Figure 18. It can be considered as a map to check the performance of the designed solution at certain distance. In general, the throughput decreases as distance increases but, the throughput reduction is more obvious for high data rates in extreme distance.

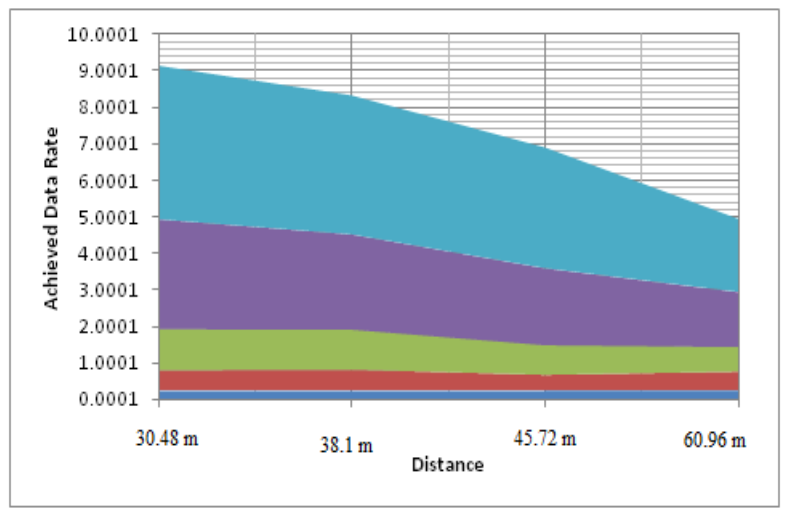

Fig. 18. Throughputs (achieved data rate) as a function of distance between Tx and $\mathrm{Rx}$ in indoor radio environment

\section{Performance Statistical Evaluation of Experimentally Collected Data}

For the two test scenarios (outdoor and indoor environments), results show the effects on performance of varying the distance on the achieved data rate for BER and the $\mathrm{Eb} / \mathrm{Neff}$. The BER, as a function of EB/Neff for each scenario, is plotted. This evaluates practically the proposed solution to validate the theory analysis as well as the simulation efforts.

The following conditions are observed from the performance evaluation:

- The throughput achieved for low rates of less than 1.2 Mbps is more stable in indoor environments. This finding can be explained by UWB pulse bounces and the reflection at the receiver side.

- The throughput for indoor environment is efficient at low-to-medium data rates ranging from $300 \mathrm{Kbps}$ to 1.2 Mbps. By contrast, for high data rates ranging from 4.8 Mbps to 9.6 Mbps, the throughput is not supportive 
during the transmission of the output power level of $12.2 \mathrm{dBm}$ (approximately $50 \%$ packet drops). At an extreme distance of 61 meters, the throughput decreases significantly, as higher data rate is more exposed to interferences and multipath effects. Also, the signal power attenuated due to the extended ranges.

- During the transmission of data at a rate of $150 \mathrm{kbps}$, BER performance is perfect, except in the extreme case wherein the distance is 61 meters. Results of the first experiment show that BER rate increases as distance increases, thus exhibiting the same trend as Eb/Neff varying according to distance.

\section{CONCLUSIONS AND Future WORKS}

IR-UWB link budget analysis can be performed as a function showing the trade-off flexibility between BER, Data rates and coverage ranges for specific IR-UWB parameters. This can be utilized for different wireless indoor scenarios and as integrator for different wireless short-ranges infrastructures.

The IR-UWB cross-layer design architecture is needed since the cost function is proposed and developed at the MAC layer level to reconfigure the physical layer parameters for optimal performance according to pre-fixed requirements (predefined Data rates, BER or ranges).

Future research can be done to optimize this solution using genetic algorithm as the cost function can be considered fitness function. The initial population started with IR-UWB certain parameters then the crossover and mutation operations are performed to approach the optimal parameters selection for optimal performance according to the fitness function (cost function) and for Pre-defined BER requirements.

\section{REFERENCES}

[1] UWB Communication Systems A Comprehensive Overview. EURASIP Book Series on Signal Processing and Communications, ed. T.K. Edited by: Maria-Gabriella Di Benedetto, Andreas F. Molisch, and C.P. Ian Oppermann, and Domenico Porcino. Vol. Volume 5, 410 Park Avenue, 15th Floor, \#287 pmb, New York, NY 10022, USA: Hindawi Publishing Corporation.

[2] Wang, Y.-q., et al., UWB multi-pulse position modulation for high datarate wireless application. The Journal of China Universities of Posts and Telecommunications, 2006. 13(4): p. 19-23.

[3] Lecointre, A., D. Dragomirecu, and R. Plana. Software defined radio layer for IR-UWB systems in Wireless Sensor Network context. in
Circuits and Systems, 2007. NEWCAS 2007. IEEE Northeast Workshop on. 2007.

[4] Aubin LECOINTRE, D.D., Robert PLANA, Design and Hardware Implementation of a Reconfigurable Mostly Digital IR-UWB Radio. ROMANIAN JOURNAL OF INFORMATION, 2008. Volume 11, Number 4: p. $295\{318$.

[5] Liu, H., et al., Taxonomy and Challenges of the Integration of RFID and Wireless Sensor Networks. IEEE network., 2008. 22(6): p. 26.

[6] Martin, R.F. Ultra-wideband (UWB) rules and design compliance issues. in Electromagnetic Compatibility, 2003 IEEE International Symposium on. 2003 .

[7] Fernandes, J.R. and D. Wentzloff. Recent advances in IR-UWB transceivers: An overview. in Circuits and Systems (ISCAS), Proceedings of 2010 IEEE International Symposium on. 2010.

[8] Pezzin, M., et al. A LDR IR-UWB Demonstration Platform for Services based on Location and Tracking. in Mobile and Wireless Communications Summit, 2007. 16th IST. 2007.

[9] Ha, D.S. and P.R. Schaumont. Replacing Cryptography with Ultra Wideband (UWB) Modulation in Secure RFID. in RFID, 2007. IEEE International Conference on. 2007.

[10] Pengyuan Yu, P.S.a.D.H.V.T., Securing RFID with Ultra-wideband Modulation: Electrical and Computer Engineering Department. Blacksburg, VA 24061

[11] Pereira, D.P., et al. Model to integration of RFID into Wireless Sensor Network for Tracking and Monitoring Animals. in Computational Science and Engineering, 2008. CSE '08. 11th IEEE International Conference on. 2008.

[12] Ze, L., S. Haiying, and B. Alsaify. Integrating RFID with Wireless Sensor Networks for Inhabitant, Environment and Health Monitoring. in Parallel and Distributed Systems, 2008. ICPADS '08. 14th IEEE International Conference on. 2008.

[13] Lâopez, T.S., et al., Integrating Wireless Sensors and RFID Tags into Energy-Efficient and Dynamic Context Networks. The Computer Journal, 2009. 52(2): p. 240-267.

[14] Beiwei, Z., H. Kunyuan, and Z. Yunlong. Network architecture and energy analysis of the integration of RFID and Wireless Sensor Network. in Control and Decision Conference (CCDC), 2010 Chinese. 2010.

[15] Baghaei-Nejad, M., et al. A Novel Passive Tag with Asymmetric Wireless Link for RFID and WSN Applications. in Circuits and Systems, 2007. ISCAS 2007. IEEE International Symposium on. 2007.

[16] Kadhim, D.J., L. Wei, and C. Wenqing. Ultra Wideband Cognitive Network Objective Issues. in Future Computer and Communication, 2009. FCC '09. International Conference on. 2009.

[17] Arslan, H. and M. Sahin, UWB-Based Cognitive Radio Networks, in Cognitive Wireless Communication Networks. 2007. p. 213-230.

[18] Di Benedetto, M.-G. and G. Giancola, Understanding ultra wide band radio fundamentals. Prentice Hall communications engineering and emerging technologies series. 2004, Upper Saddle River, N.J.: Prentice Hall PTR. 